\title{
Arthroscopic Repair of Massive Cuff Tears With Large Subscapularis Tendon Ruptures (Lafosse III/IV): A Prospective Magnetic Resonance Imaging-Controlled Case Series of 26 Cases With a Minimum Follow-up of 1 Year
}

Grueninger, Patrick ; Nikolic, Nikola ; Schneider, Joerg ; Lattmann, Thomas ; Platz, Andreas ; Chmiel, Corinne ; Meier, Christoph

\begin{abstract}
PURPOSE To prospectively assess arthroscopic repair of massive cuff tears (MCT) in a highly selective patient group with large subscapularis (SSC) tendon tears by means of clinical results and magnetic resonance imaging (MRI) studies. METHODS Between April 2009 and December 2010,26 patients with MCT were treated with arthroscopic rotator cuff repair. Only lesions involving a large tear of the SSC tendon (Lafosse III or IV) in combination with a complete tear of the supraspinatus (SSP) tendon and a tear of at least the anterior third of the infraspinatus (ISP) tendon were included. Minimum follow-up was 1 year. Pre- and postoperative assessment included a standardized clinical examination, subjective patient outcome, and MRI (structural integrity, fatty muscle infiltration, and muscular mass). RESULTS Mean follow-up was 17 months (range, 12 to 34 months). MRI was performed in 25 patients. In $21(84 \%)$ the cuff repair was intact. A partial retear of the SSC was found in 2 patients (8\%). In 2 patients $(8 \%)$ a full-thickness retear of the posterosuperior cuff was observed (1 SSP, 1 SSP/ISP). A significant increase of the muscle mass and decrease of fatty infiltration was observed for the SSC and SSP but not for the ISP. The mean Constant-Murley score improved from 36 to 86 points $(\mathrm{P}<.001)$ with all its subscores as well $(\mathrm{P}<.001)$. Muscular strength improved for the SSC $(4.9 \mathrm{v} 3.0, \mathrm{P}<.001)$, SSP $(4.6 \mathrm{v} 2.9, \mathrm{P}<.001)$, and ISP $(4.8 \mathrm{v} 3.4, \mathrm{P}<.001)$. Overall patient satisfaction was high $(3.6$ $\pm 0.8)$. CONCLUSIONS Arthroscopic repair of MCT involving the ISP, SSP, and large tears of the SSC provides a reliable tendon healing, in particular for the SSC tendon, combined with good functional results. LEVEL OF EVIDENCE Level IV, therapeutic case series.
\end{abstract}

DOI: https://doi.org/10.1016/j.arthro.2015.05.012

Posted at the Zurich Open Repository and Archive, University of Zurich

ZORA URL: https://doi.org/10.5167/uzh-116943

Journal Article

Accepted Version

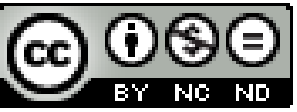

The following work is licensed under a Creative Commons: Attribution-NonCommercial-NoDerivatives 4.0 International (CC BY-NC-ND 4.0) License.

Originally published at: 
Grueninger, Patrick; Nikolic, Nikola; Schneider, Joerg; Lattmann, Thomas; Platz, Andreas; Chmiel, Corinne; Meier, Christoph (2015). Arthroscopic Repair of Massive Cuff Tears With Large Subscapularis Tendon Ruptures (Lafosse III/IV): A Prospective Magnetic Resonance Imaging-Controlled Case Series of 26 Cases With a Minimum Follow-up of 1 Year. Arthroscopy: The Journal of Arthroscopic Related Surgery, 31(11):2173-82.

DOI: https://doi.org/10.1016/j.arthro.2015.05.012 
8 Arthroscopic Repair of Massive Rotator Cuff Tears with Large Subscapularis Tendon

9 Ruptures (Lafosse III/ IV): A Prospective Magnetic Resonance Imaging-Controlled 
14 Purpose: The purpose of this study was to prospectively assess arthroscopic repair of massive cuff tears (MCT) in a highly selective patient group with large subscapularis (SSC) tendon tears by means of clinical results and magnetic resonance imaging (MRI) studies. Methods: Between April 2009 and December 2010, 26 patients with MCT were treated with arthroscopic rotator cuff repair. Only lesions involving a large tear of the SSC tendon (Lafosse III or IV) in combination with a complete tear of the supraspinatus (SSP) tendon and a tear of at least the anterior third of the infraspinatus (ISP) tendon were included. Minimum follow-up was 1 year. Pre- and postoperative assessment included a standardized clinical examination, subjective patient outcome and MRI (structural integrity, fatty muscle infiltration and muscular mass).

Results: Mean follow-up was 17 months (range 12-34 months). MRI was performed in 25 patients. In 21 (84\%) the cuff repair was intact. A partial re-tear of the SSC was found in 2 (8\%). In 2 patients (8\%) a full thickness re-tear of the posterosuperior cuff was observed (1 SSP, 1 SSP/ISP). A significant increase of the muscle mass and decrease of fatty infiltration was observed for the SSC and SSP but not for the ISP. The mean Constant-Murley score improved from 36 to 86 points $(P<0.001)$ with all its subscores as well $(p<0.001)$. Muscular strength improved for the SSC (4.9 vs. $3.0, p<0.001)$, SSP (4.6 vs. $2.9, p<0.001)$, and ISP (4.8 vs. $3.4, p<0.001)$. Overall patient satisfaction was high $(3.6 \pm 0.8)$.

Conclusions: Arthroscopic repair of MCT involving the ISP, SSP and large tears of the SSC provides a reliable tendon healing in particular for the SSC tendon combined with good functional results. 
Massive rotator cuff tears (MCT) may be associated with pain, disability and loss of active range of motion. In some cases pseudoparalysis may be observed. Involvement of three tendons is associated with a worse functional impairment compared to patients with a tear of two tendons only ${ }^{1}$. If untreated, MCT can lead to rotator cuff arthropathy and instability. The etiology of MCT is degenerative, traumatic or a combination of both. The structure results of arthroscopic MCT repair show re-tear rates between $16 \%$ and $53 \%{ }^{2-4}$. However, comparison of the literature may be difficult because different definitions of MCT are commonly used. Some authors favor the Cofield classification ${ }^{5}$ defined as a tear of greater than five centimeters in diameter. Others use the definition established by Gerber and coworkers ${ }^{1}$ defined as detachment of at least two entire tendons or the Patte classification system ${ }^{6}$ which differentiates three stages (stage I: proximal stump close to bony insertion; stage II: proximal stump at level of humeral head; and stage III: proximal stump at level of glenoid). Others do not provide any classification at all ${ }^{7}$. Furthermore, the surgical techniques as described in the literature differ widely ${ }^{2,8,9}$. In a previous study it was demonstrated that arthroscopic repair of isolated traumatic large SSC tendon tears achieves excellent results regarding function and tendon healing ${ }^{10}$.

The purpose of this study was to prospectively assess arthroscopic repair of MCT in a highly selective patient group with large subscapularis (SSC) tendon tears by means of clinical results and magnetic resonance imaging (MRI) studies. Our hypothesis was that repair of SSC in the situation of a MCT provides a reliable tendon healing in particular for the SSC tendon combined with good functional results. However, the functional and structural results may be inferior compared to isolated SSC tears.

61

\section{Methods}


All patients who underwent arthroscopic rotator cuff repair Between between January 2008 and December 2010 were identified.,- Only patients 26 consecutive patients (18 men and 8 women) with MCT involving a large tears of the SSC tendon in combination with a complete full-thickness tear of the supraspinatus (SSP) tendon and a full-thickness tear of at least the anterior third of the infraspinatus (ISP) tendon undergoing arthroscopic rotator cuff repair were included in this prospective case series. The SSC tendon tears were classified according to Lafosse ${ }^{11}$ (type I: partial lesion of the superior one-third; type II: complete lesion of the superior one-third; type III: complete lesion of the superior two-thirds; type IV lesion: complete tendon lesion with well-centered humeral head and fatty infiltration $\leq$ Goutallier $3^{12}$; type V: complete tendon lesion with subluxated humeral head, coracoid conflict and fatty infiltration $\geq$ Goutallier 3). Only large SSC lesions (Lafosse $\geq \mathrm{III}$ ) were included. Patients with isolated SSC or with minor tears of the SSC only were excluded. Anterosuperior cuff tears without involvement of the ISP were excluded as well. Standardized clinical and radiological assessments were performed at the same time preoperatively and at least one year after operation. Patients who had their surgery before 2010 had a longer follow-up because the clinical examinations and MRI were not initiated before 2011 for study purposes. Preoperative pseudoparalysis, return to work, return to sports, patient satisfaction, intra- and postoperative complications, and re-interventions were recorded as well. The study was approved by the local ethics committee. Informed consent for operative treatment and all the follow-up investigations was obtained from all the patients.

\section{Operative technique}

87 All operations were performed by the same surgeon. The arthroscopic procedures were performed with patients under general anesthesia with an interscalene catheter for postoperative pain control. The patient was seated in a standardized beach-chair position

90 with arm traction of 2 to $3 \mathrm{~kg}$. Perioperative antibiotic prophylaxis with cefuroxime was 
routinely administered. A standard $30^{\circ}$ arthroscope was used. According to Lafosse et al. ${ }^{11}$, the portals were named from A (posterior "soft spot" portal) to $E$ (anterosuperior portal). In cases with significant retraction of the SSC an additional portal for a traction suture was created (J portal). Before the arthroscope was introduced into the glenohumeral joint through portal $A$, the joint was infiltrated with $20 \mathrm{~mL}$ of diluted epinephrine $(1 \mathrm{ml}$ of epinephrine $[1 \mathrm{mg} / \mathrm{mL}]$ and $19 \mathrm{~mL}$ of normal saline solution) to decrease intraoperative bleeding.

Furthermore, systolic blood pressure was maintained below $100 \mathrm{mmHg}$ during surgery. With these measures, the intra-articular pressure could be kept as low as $35 \mathrm{~mm} \mathrm{Hg}$ until the end of the intervention for most patients. In some cases the pressure had to be increased in a stepwise manner during the procedure to provide good visibility. However, a continuous maximum pressure of $60 \mathrm{~mm} \mathrm{Hg}$ was never exceeded in this series.

The diagnostic arthroscopy was performed, and a probe was inserted through portal D (antero-lateral portal). The tear of the SSC was classified according to Lafosse ${ }^{11}$. To assess the true extent of the posterosuperior (PSUP) cuff tear the scope was then placed in the lateral C portal after subacromial bursectomy. For the tenodesis of the long head of the biceps tendon (LHBT), the first suture of a double-loaded threaded suture anchor was used to perform a "lasso-loop stich" ${ }^{13}$. The second suture of this anchor was used for reconstruction of the most superior part of the SSC tendon at a later stage. The surgical technique for the repair of the SSC has previously been described in detail ${ }^{10}$. In general the reconstruction of the SSC was performed with two threaded suture anchors usually with application of U-stitches. The remaining suture of the LHBT tenodesis was used for a lassoloop stitch at the upper border of the SSC, achieving a pseudo-double-row reconstruction (Fig 1).

The coracohumeral distance is defined as the shortest distance from the tip of the coracoid to the humeral head on axial MRI scans (taken from the cut that best images the base of the coracoid) ${ }_{4}^{14}$. According to Gerber et al. ${ }^{15}$, a narrowing $<6 \mathrm{~mm}$ may be considered 
pathological which may cause subcoracoidal impingement signs such as dull pain in the anterior aspect of the shoulder when the shoulder is in a forward flexed, adducted and $\underline{\text { internally rotated position. No coracoplasties were performed in the study group since neither }}$ a pathological narrowing of the coracohumeral distance nor clinical signs of subcoracoidal impingement were observed in any of our patients. because we did not see any signs of subcoracoid impingement (dull pain in the anterior aspect of the shoulder when the shoulder is in a forward flexed, adducted and internally rotated position).

If required a proper release of the PSUP cuff including capsulotomy, bursectomy and dissection to as far as to the spina scapulae were performed. The footprint of the SSP and the ISP were prepared once again with a bur. The PSUP cuff was repaired from posterior to anterior. A double-row suture bridge technique was routinely performed for U-shaped tears for a complete restoration of the footprint ${ }^{1416}$. Depending on the size of the tear, either two or three double-loaded threaded anchors were applied for the medial suture row. The sutures were passed through the cuff in a horizontal mattress configuration and tied. For cleavage tears, a deep-layer lasso-loop (Fig 2A and B) was placed in the deep layer and the ends were then passed through the superficial layer and tied similar to the technique described by Toussaint et al ${ }^{1517}$. One tail of each suture was anchored laterally in the greater tuberosity using two knotless suture anchors. In all patients an acromioplasty was routinely performed. In symptomatic patients an AC-resection was done at the end of the procedure.

\section{Rehabilitation}

Postoperatively all patients were immobilized with a $45^{\circ}$ abduction pillow for 6 weeks.

Physiotherapy was initiated on day one consisting of pain-free passive range of motion only. External rotation was limited to $0^{\circ}$ and internal rotation to $45^{\circ}$. Patients were encouraged to perform active wrist and elbow movements from the beginning. For personal hygiene, all patients were instructed and advised to shower with a waterproof abduction wedge. After 6 weeks, active exercises were started to regain full range of motion. Strengthening exercises 
were allowed after 12 weeks. A gradual return to sports activities was allowed, depending on the kind of sport, but not before 6 months, and overhead sports not before 9 months postoperatively.

\section{Clinical evaluation}

Preoperatively, the patient's history, the history of injury or the onset of symptoms in cases without previous trauma was registered. A standardized physical examination was performed before the operation and at follow-up by the first author (P.G.). The SSC function was tested with the modified belly-press test and the modified lift-off test as described by Lafosse et al. 11. The SSP function was tested by measuring abduction strength with the patient standing, the arm abducted to 90 degrees in the scapular plane, the elbow extended, and the forearm pronated. The resistance was applied at the wrist and the strength measured by using a dynamometer. The ISP external rotation strength was measured at 0 degrees abduction and 0 degrees external rotation. Muscular strength was graded from 0 to 5 according to the classification of neurologic assessment. Pseudoparalysis was defined according to Werner ${ }^{16}$ ${ }_{18}$ as an active forward elevation of less than 90 degrees in association with full passive motion. The clinical examination included the Constant-Murley score (CMS) ${ }^{1719}$. At follow-up, our patients were also asked to rate their level of satisfaction as poor (1 point), fair (2 points), good ( 3 points) and excellent (4 points) and were questioned on their return to work and sports activities.

\section{Radiological evaluation}

All patients underwent a standardized radiographic evaluation including a true anteroposterior radiograph in neutral rotation and an axillary view preoperatively and immediately after surgery. Preoperatively, all patients were also evaluated with contrast-enhanced magnetic resonance imaging (arthro-MRI with gadolinium). The SSC tears were classified 
according to Lafosse ${ }^{11}$. The Patte classification ${ }^{6}$ was used for tears of the SSP and ISP (stage I: the proximal stump is close to the insertion; stage II: the stumps lies at the level of the humeral head; and stage III: the stump is seen at the level of the glenoid). Fatty infiltration of the SSC, SSP and ISP was graded according to Goutallier et al., ${ }^{12}$ modified by Fuchs et al. ${ }^{18}{ }^{20}$ for MRI, from 0 to 4 . Accordingly, grade 0 indicates no fatty infiltration; grade 1 some fatty streaks; grade 2 less fat than muscle; grade 3 , as much fat as muscle; and grade 4, more fat than muscle. The cross sectional area (CSA) of the SSC, SSP and ISP was measured according to the method proposed by Zanetti et al. ${ }^{19}{ }^{21}$ using standard measurement tools in our picture archiving and communicating system software. The CSA was measured in square millimeters on the most lateral image on which the scapular spine was in contact with the rest of the scapula in the sagittal reconstructions.

At follow-up, a native MRI investigation with a dedicated shoulder coil (Magnetom Avanto, 1,5T; Siemens Medical Solution, Erlangen, Germany) without contrast enhancement was obtained. On the axial and paracoronal views, the tendons of SSC, SSP and ISP were evaluated regarding continuity and retraction. Tendon integrity was assessed on axial, paracoronal and sagittal T2-weighted and proton density-weighted sequences (Fig $3 \mathrm{~A}-\mathrm{C}$ ). Integrity of the cuff repair was assessed as follows: normal tendon, intratendinous signal abnormality, gap in a tendon filled with a water-equivalent signal, and complete rupture of repair. A repair with either a gap or a complete rupture was considered as a failed repair. Intratendinous signal abnormalities with a water-equivalent signal within the footprint were classified as intact because this finding was likely the result of fluid traveling the suture line which may persist for a long time postoperatively ${ }^{20,2122,23}$. The fatty muscular infiltration and CSA of the SSC SSP and ISP were determined as described earlier. The integrity of the LHBT was evaluated on the most superior axial cross section where the bicipital groove was still visible. In contrast to the preoperative MRI studies, only native MRI studies were performed at follow-up. Acceptance of an invasive technique for purely scientific reasons 
without direct benefit to the patient may be low and may be controversial because of ethical reasons. Furthermore, the superiority of MR arthrography for evaluation the structural integrity of cuff repairs is not proven for complete re-tears and its diagnostic performance for partial-thickness re-tears appears to be limited as well ${ }^{21} \underline{23}$. The application of intra-articular gadolinium does not change the appearance of the muscles on the images. Thus, direct comparison of fatty degeneration in arthro- and native MRI is not compromised. The complete preoperative and postoperative radiologic assessment was performed by an experienced MR radiologist with special training in musculoskeletal imaging. This radiologist was blinded to the clinical results.

\section{Statistical analysis}

The results were analyzed with statistical software (IBM SPSS, version 20; IBM, Armonk, NY and Stata version 13.1, StataCorp LP, Texas). Statistical analysis for dependent groups was performed with the paired $t$ test in case of parametric data and the Wilcoxon singed rank test in case of non-parametric numerical data. Categorical between group analyses were performed by means of Chi-squared test. All data are presented as means and standard deviations, with ranges in parentheses. A double-sided $\mathrm{P}<0.05$ was considered statistically significant.

\section{Results}

\section{Baseline patient characteristics}

\section{Twenty-six patients (18 men and 8 women) were included in this therapeutic case series.}

The mean age of the patients was $64 \pm 8$ years (range, 46 to 75 years). The dominant shoulder was affected in 19 patients (73\%). In 21 (81\%) patients a shoulder injury was recorded in the history. Seven patients (33\%) sustained a high velocity trauma and 14 (67\%) 
patients a fall from standing height. In 6 patients (28\%) the trauma mechanism included an anterior shoulder dislocation which was treated by closed reduction in all patients. Only 5 (19\%) patients complained about shoulder problems unrelated to an adequate a previous injury. These MCTs were rated as degenerative tears. Pseudoparalysis was seen in 10 (38\%) patients. Before trauma and/or onset of symptoms 14 (54\%) of patients were working, $11(42 \%)$ were retired and $1(4 \%)$ was already disabled before surgery unrelated to his shoulder problems. The mean interval from trauma to surgery was $3.1 \pm 2.9$ months (range, 0.9-12.2 months). The mean duration of symptoms in degenerative cases was $28.8 \pm 39.1$ months (range 5.0- 96.7 months).

\section{Intraoperative findings}

The arthroscopic evaluation of the 26 patients resulted in 15 type III SSC lesions (58\%) and $11(42 \%)$ type IV lesions. The SSP tendon retraction as classified according to Patte showed $4(15 \%)$ grade I lesions, 19 (73\%) grade II lesions and $3(12 \%)$ grade III lesions. In 4 patients (15\%), only the anterior third of the ISP tendon was affected, in 22 patients (85\%) a complete tear of the ISP was observed. Retraction of the ISP was classified as grade I in 11 patients (42\%), grade II in $10(38 \%)$ and grade III in $1(4 \%)$ case, respectively. In 7 patients $(27 \%)$ the LHBT was completely dislocated and a pronounced anterior instability was observed in 6 cases $(23 \%)$. Four (15\%) LHBT were absent and $9(35 \%)$ were of a tendinopathic appearance with a partial rupture of less than $50 \%$. In summary, none of our patients presented without any LHBT pathology.

\section{Follow-up}

Mean follow-up was 17 months (range 12-34 months). However, 1 patient with an excellent clinical result refused MRI control. Thus, a complete follow-up including clinical examination and MRI investigation was available in 25 patients (96\%). Two out of 14 working patients 
(14\%) had to change their previous work due to their shoulder problems and 2 heavy workers $(14 \%)$ were unable to return to work. Eleven patients were already retired at the time of their operation, and 1 patient was already disabled. Fifteen patients had performed different kinds of recreational sports prior to their shoulder problems. At follow-up 12 patients $(80 \%)$ of this subgroup returned to the same level of their previous activities whereas 3 patients $(20 \%)$ had to reduce their level due to persistent shoulder impairment. Overall, the level of patient satisfaction was $3.6 \pm 0.8$ (range $2-4$ ) at follow-up. Twenty patients $(77 \%)$ rated their outcome as excellent, 2 patients $(8 \%)$ had a good result, and 4 patients $(15 \%)$ were satisfied. We found no statistical difference between the two subgroups (with and without retears) regarding the level of satisfaction (3.0 vs. $3.7, p=0.106)$.

\section{Clinical outcome}

The results of the clinical examination are shown in Table 1. Preoperatively all patients were able to perform the belly-press test but muscular strength was reduced compared to the healthy contralateral side. Twelve patients (46\%) were unable to demonstrate a correct lift-off test because of limited internal rotation or pronounced pain. The remaining 14 (54\%) patients presented all with a positive lift-off test. In contrast, at follow-up, all patients correctly performed both tests and a significant improvement of strength in these specific SSC tests was found. Prior to surgery, all but one patient (4\%) were able to demonstrate a correct abduction strength test for the SSP. At follow-up, all patients were able to perform the test and an improvement of strength was recorded. In accordance with the other tests, ISP external rotation strength was significantly improved. Pseudoparalysis was not observed in any patient at follow-up. The CMS and all its subscores- pain, activities of daily living, range of motion, and strength-showed a significant improvement at follow-up compared with the preoperative situation. The CMS of the subgroup with re-tears $(n=4)$ only showed a trend for statistical significance $(p=0.068)$ at follow-up compared with the preoperative score whereas the improvement of the CMS in the subgroup with an intact structural integrity of the footprint 
$(n=21)$ was statistically significant $(p<0.001)$. However, we only found a trend for the

278 difference of the CMS at follow-up between the two subgroups $(p=0.055)$. Failure of LHBT

279 tenodesis $(n=3)$ did not affect clinical outcome.

Complications were observed in 3 patients (12\%). An acute joint infection caused by proprionibacterium acnes 3 weeks after the initial surgery was successfully treated with arthroscopic lavage and antibiotics (levofloxacin/rifampicin) for 3 months. Another patient underwent revision arthroscopy for a painful cuff capture 9 months after rotator cuff repair. Despite intraoperative sampling, no bacteria were detected. In 1 patient a mesacromion was not surgically addressed when the rotator cuff was repaired. At follow-up, the patient presented with pain and a poor functional result (CMS 50 points). MR imaging revealed a large re-tear of the SSP and ISP (Patte III). Open latissimus dorsi transfer combined with acromial fusion was performed.

\section{MRI follow-up}

Complete structural integrity of the footprint of the SSC, SSP and ISP was observed in 17 (68\%) patients out of $25 \mathrm{MRI}$ investigations at follow-up. Some intratendinous signal abnormalities were seen in 4 cases ( 3 SSP, 1 SSP+ISP). However, this was also graded as intact. Thus, the cuff repair was considered intact in a total of 21 patients (84\%). A fullthickness re-tear of the superior one-third of the SSC tendon (Lafosse type II) was evident in 2 cases (8\%) (Fig 4A and B). However, these re-tears of the SSC were smaller than the initial tear. In 1 patient (4\%) the MRI showed a failed repair with a complete rupture of the SSP. A repair failure of the posterosuperior cuff (SSP and ISP), exceeding the size of the initial tear, was seen in 1 case (4\%). If intratendinous signal abnormalities were also 
SSC, $24 \%$ for the SSP and $8 \%$ for the ISP. All humeral heads were anatomically centered in the glenoid, even in patients with repair failures.

The alterations of the muscular mass and the course of fatty infiltration of the rotator cuff are shown in Table 2. Neither a change of the muscle mass nor fatty infiltration was seen for the ISP whereas a significant increase of the CSA and improvement of muscle quality was observed for the SSC and SSP. Follow-up MRI also showed an intact LHBT tenodesis in 19 out of 22 cases. Thus, the failure rate for the tenodesis was $14 \%$.

\section{Discussion}

Arthroscopic repair of MCT with large SSC tears was associated with a significant improvement of shoulder function and high patient satisfaction. MRI at follow-up showed an intact rotator cuff repair in $84 \%$. The re-tear rate was $8 \%$ for the SSC and equally for the PSUP cuff. A significant increase of the muscle mass and decrease of fatty infiltration was observed for the SSC and SSP, but not for the ISP.

\section{Clinical outcome}

Arthroscopic repair of MCT is known to be associated with a good clinical result regarding pain, motion and functional outcome scores ${ }^{2,3,4,8}$. The analysis of our study group demonstrated a significant improvement of the overall CMS and all its subscores as well. The CMS increased from 36 points preoperatively to 86 points at 1 -year follow-up. This is in-line with other investigators. Kim reported an improvement from an absolute CMS of $45-$ to 75 points ${ }^{4}$. However, they also included 2 -tendon tears in their study group, but similar to our investigation, they applied a suture-bridge technique for the tendon repair. Other studies showed similar CMS at follow-up ${ }^{2,3}$. In the current study, only a statistical trend but no significant difference was found for functional outcome between the patients with re-tears 
and those with complete tendon healing. Interestingly, our study group presented with a high level of patient satisfaction at follow-up with no difference between the two subgroups. An improved clinical outcome even in patients with re-tears after arthroscopic rotator cuff repair was reported in other studies ${ }^{2,4}$. However, the healed groups were significantly better than the re-tear groups in both studies.

The rotator cable is an important structure within the avascular zone of the rotator cuff which spans crescent-like from the upper half of the SSC insertion to the inferior border of the ISP insertion at the humerus. A tear of at least the upper half the SSC tendon is associated with a disruption of the anterior attachment of the rotator cable $23 \underline{25}$. Accordingly, a posterior disruption of the rotator cable attachment was defined as a tear that involved $100 \%$ of the ISP insertion. According to the concept of the "suspension bridge" and the observations by Denard et al. ${ }^{23,2425,26}$ may occur if at least one rotator cable attachment is disrupted. Furthermore, this concept also explains why patients with a tear inside the crescent and an intact rotator cable demonstrate good function.

\section{Structural integrity of the tendon repair}

In the current analysis the rate of re-tears of the SSC tendon repair was higher compared to our previous study in which only isolated large SSC lesions (Lafosse III/IV) were prospectively evaluated ${ }^{12}$. This may be attributed to the accompanying tears of the PSUP cuff since the time interval between onset of symptoms and operation and the surgical technique of the SSC repair were identical. Our SSC re-tear rate failure rate of $8 \%$ was well within the range of other series published in the current literature. In a recent study, Toussaint et al. ${ }^{25}-27$ reported a re-tear rate of $8 \%$ in 129 patients with arthroscopic repair of either isolated SSC tears or in combination with limited anterosuperior tears with a radiologic follow-up of at least 6 months. In other studies which all used either CT arthrography or MRI 
to assess structural integrity of the SSC repair, re-tear rate ranged from 5-14\% ${ }^{11,26-2828-30}$. Lanz et al. ${ }^{29}{ }^{31}$ analyzed the arthroscopic repair of 46 large_SSC tears including a radiologic follow-up of 2-4 years. In only 23 patients the SSP and ISP were involved as well. Although detailed results regarding the SSC are provided with an overall re-tear rate of $11 \%$ the study lacks information about the radiologic definition of a re-tear and potential differences between different tear patterns such as an isolated rupture or a MCT. The authors did not find any influence of the interval between onset of symptoms and surgery when analyzing a cut-off of 6 months.

Overall, our re-tear rate after MCT repair was $16 \%$ when intratendinous signal abnormalities on the follow-up MRI were considered as intact repairs ${ }^{20-2122,23}$. However, comparison of different studies may be hazardous since the definition of a re-tear is not uniform. Some examiners used the Sugaya classification ${ }^{22}{ }_{2}{ }_{-}$or the classification proposed by Lafosse ${ }^{2}$, ${ }^{2022}$. Others performed MRI or CT arthrography without providing specific information on the definition of a re-tear ${ }^{4,2931}$. There seems to be no consensus regarding the definition of a retear on MRI. According to Sugaya's classification, a minor discontinuity is considered a retear $^{2224}$. In contrast, Lafosse argues that intratendinous leakage is consistent with a small amount of fluid travelling down the suture and not a partial re-tear ${ }^{2022}$. By applying those definitions on our findings, our SSP re-tear rate differs from 8-to $24 \%$. However, a complete healing with intact structural integrity of all tendon repairs was found in $72 \%$ of our patients. Focusing on the SSC repair, a complete structural integrity of the footprint was observed in 92\%. Lanz, using a similar technique as described in the current work for the reconstruction of the SSC footprint, reported a re-tear rate of $11 \%$ for the SSC ${ }^{2931}$. However, this rate also includes isolated SSC repairs. In only $50 \%$ the cuff tear involved the SSP and the ISP as well.

\section{Muscle alterations}


Kim et al. found that the preoperative degree of fatty infiltration of the SSP and ISP was significantly higher in patients with re-tears after MCT repair ${ }^{4}$. Interestingly, no difference was observed for the SSC. They also found that the preoperative extent of retraction and preoperative degree of fatty infiltration of the ISP were predictive factors for re-tears. In another study, fatty infiltration of the SSC did not progress after successful repair of SSC lesions Lafosse III and IV in the vast majority of the patients. Of interest, a recovery of fatty infiltration was not seen in any patient ${ }^{2931}$. Others reported a progression of fatty infiltration despite successful SSC repair ${ }^{25,2827,30}$. Gerber et al found a recovery of muscle atrophy in successful repaired SSP tendons but neither in the ISP nor SSC after open repair of MCT 3032. A progression of fatty infiltration in all three muscles was observed. Our findings are in contrast to most of the above mentioned studies. A significant increase of the muscular mass and decrease of fatty infiltration was observed for the SSC and SSP whereas no changes were noted for the ISP. In a previous study, muscular alterations after repair of isolated large SSC tears were investigated ${ }^{10}$. In that study, the muscular mass increased in all three muscles but only the SSC demonstrated a decrease of fatty infiltration. However, the assessment of fatty degeneration is known to be associated with a relatively low reliability ${ }^{31} \underline{33}$. Furthermore, interpretations of the Goutallier grade and muscular mass based on a preoperative MRI may be hazardous. In a remarkable study by Jo and co-workers, preoperative MRI was compared with MRI undertaken 3 days after arthroscopic subacromial decrompression or instability surgeries ${ }^{32} \underline{34}$. No differences were found for the CSA of the SSP and ISP and grading of fatty infiltration of all the examined muscles. A significant increase of the CSA of the SSC was observed although no rotator cuff surgery was performed. The authors concluded that excessive positional vulnerability of the SSC may be responsible for this finding. In a second step, preoperative- and postoperative MRI after rotator cuff surgery were analyzed. Rotator cuff surgery changed the Goutallier grade for the SSP and the ISP but not for the SSC. Regarding CSA, the SSP increased by $45 \%$ and the ISP by $12 \%$ for massive tears, respectively. Considering these findings, our results 
increased muscle mass and improved muscle quality but mere the result of volumetric

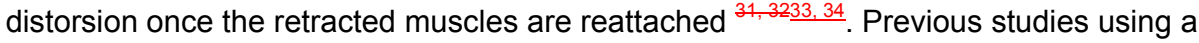
similar study design as ours may be affected as well.

\section{Limitations}

415 A limitation of our study is the single surgeon technique. There is also a potential bias due to the fact that all clinical examinations preoperatively and at follow-up were performed by the same surgeon. Furthermore, our minimum follow-up of 1 year is rather short. However,

418 Mihata et al. ${ }^{9}$ evaluated structural integrity of the footprint after arthroscopic cuff repair in

419201 patients up to 24 months after different techniques of repair by repetitious MRI. In their subgroup of 60 shoulders with large to massive cuff tears a re-tear was found in 12 shoulders (20\%) but no detailed information is given for the SSC. After 2 years, only one more re-tear was seen. When the suture-bridge technique was separately analyzed, no retear was seen later than 6 months postoperatively. These observations may support the view that a follow-up of 1 year is reliable to study the structural integrity of rotator cuff repair.

As we did not perform MRI right after cuff repair we do not have a reliable baseline investigation regarding muscular mass and fatty infiltration.

The sample size of this case series $(n=26)$ is rather small, we did not have a control group and our study is underpowered from a statistical view. However, we have applied strict inclusion criteria for this study by investigating only MCT with significant involvement of the SSC (Lafosse III and IV). Only a few studies were found in the literature addressing arthroscopic repair of MCT which included a radiological follow-up similar to our study design. Choi et al studied 258 patients but only 15 patients had massive tears $(>5 \mathrm{~cm})$ and no 
details were provided regarding involvement of the SSC ${ }^{3}$. In another study, only 19 patients

436 out of 154 were classified as massive tears (Patte III) ${ }^{2}$. Kim reported the results of suture-

437 bridge repair in 66 patients with $\mathrm{MCT}^{4}$. The authors also included 2-tendon tears and only

43826 patients were reported to have a SSC tear without any details provided regarding the 439 grade of SSC involvement.

\section{Conclusions}

443 Arthroscopic repair of MCT involving the ISP, SSP and large tears of the SSC provides a

444 reliable tendon healing in particular for the SSC tendon combined with good functional 445 results. 
1. Gerber C, Fuchs B, Hodler J. The results of repair of massive tears of the rotator cuff. J Bone Joint Surg Am 2000; 82: 505-515.

2. Toussaint B, Schnaser E, Bosley J, Lefebvre Y, Gobezie R. Early structural and functional outcomes for arthroscopic double-row transosseous-equivalent rotator cuff repair. Am J Sports Med. 2011;39:1217-25.

3. Choi S, Kim MK, Kim GM, Roh YH, Hwang IK, Kang H. Factors associated with clinical and structural outcomes after arthroscopic rotator cuff repair with suture bridge technique in medium, large, and massive tears. J Shoulder Elbow Surg 2014; 23: 1675-1681.

5. Cofield RH. Rotator cuff disease of the shoulder. J Bone Joint Surg Am 1985;67:974979. 
7. Ji JH, Shafi M, Jeong JJ, Park SE. Arthroscopic repair of large and massive rotator cuff tears using the biceps incorporating technique: mid-term clinical and anatomical results. Eur J Orthop Surg Traumatol 2013; Oct 2 (Epub ahead of print)

8. Denard PJ, Jiwani AZ, Laedermann A, Burkhart SS. Long-term outcome of arthroscopic massive rotator cuff repair: the importance of double-row fixation. Arthroscopy 2012; 28: 909-915..

9. Mihata T, Watanabe C, Fukunishi K, Ohue M, Tsujimura T, Fujiwara K, Kinoshita M. Functional and structural outcomes of single-row versus double-row versus combined double-row and suture-bridge repair for rotator cuff tears. Am J Sports Med. 2011; 39:2091-2098.

10. Grueninger P, Nikolic N, Schneider J, Lattmann T, Platz A, Chmiel C, Meier C. Arthroscopic repair of traumatic isolated subscapularis tendon lesions (Lafosse Type III or IV): A prospective magnetic resonance imaging-controlled case series with 1 year follow-up. Arthroscopy 2014; 30: 665-672.

11. Lafosse L, Jost B, Reiland Y, Audebert S, Toussaint B, Gobezie R. Structural integrity and clinical outcomes after arthroscopic repair of isolated subscapularis tears. J Bone Joint Surg Am 2007; 89: 1184-1193. degeneration in cuff ruptures. Pre- and postoperative evaluation by CT scan. Clin Orthop Relat Res 1994; 304:78-83. 

shoulder. Clin Orthop Relat Res 1987; 214: 160-164.

Formatiert: Schriftartfarbe: Schwarz, Französisch (Schweiz)

\section{Tan V, Moore RS Jr, Omarini L, Kneeland JB, Williams GR Jr, lannotti JP. Magnetic} $\underline{\text { resonance imaging analysis of coracoid morphology and its relation to rotator cuff }}$ tears. Am J Orthop (Belle Mead NJ) 2002; 31(6):329-333.

15. Gerber C, Terrier F, Zehnder R, Ganz R. The subcoracoid space. An anatomic study. Clin Orthop Relat Res 1987; 215:132-138. 13. 14.16. Bales C, Andersen K. Arthroscopic double-row repair of full-thickness rotator cuff tears using a suture bridge technique. Oper Tech Sports Med 2007; 14: 144-149.

\subsection{Toussaint B Schnaser E, Lafosse L, Bahurel J, Gobezie R. A new approach to} improving the tissue grip of the medial-row repair in the suture-bridge technique: the “modified lasso-loop stitch". Arthroscopy 2009: 25:691-695.

\subsection{Werner CM, Steinmann PA, Gilbart M, Gerber C. Treatment of painful} pseudoparesis due to irreparable rotator cuff dysfunction with the Delta III reverseball-socket total shoulder prosthesis. J Bone Joint Surg Am 2005; 87: 1476-1486.
Formatiert: Schriftart: Schriftartfarbe: Schwarz, Französisch (Schweiz)

Formatiert: Einzug: Links: $1.27 \mathrm{~cm}$, Keine Aufzählungen oder Nummerierungen

Formatiert: Keine Aufzählungen oder Nummerierungen

Formatiert: Englisch (USA)

Formatiert: Einzug: Links: $0 \mathrm{~cm}$ 
18.20. Fuchs B, Weisshaupt D, Zanetti M, Hodler J, Gerber C. Fatty degeneration of the muscles of the rotator cuff: Assessment by computed tomography versus magnetic resonance imaging. J Shoulder Elbow Surg 1999; 8: 599-605.

19.21. Zanetti M, Gerber C, Hodler J. Quantitative assessment of the muscles of the rotator cuff with magnetic resonance imaging. Invest Radiol 1998; 33:163-170.

\subsection{Lafosse L, Brozska R, Toussaint B, Gobezie R. The outcome and structural} integrity of arthroscopic rotator cuff repair with use of the double-row suture anchor technique. J Bone Joint Surg Am 2007; 89: 1533-151.

\subsection{Duc SR, Mengiardi B, Pfirrmann CW, Jost B, Hodler J, Zanetti M. Diagnostic} performance of MR arthrography after rotator cuff repair. Am J Roentgenol 2006; 186: 237-241.

\subsection{Sugaya H, Maeda K, Matsuki K, Moriishi J. Functional and structural outcome} after arthroscopic full-thickness rotator cuff repair: single-row versus dual-row fixation. Arthroscopy 2005; 21: 1307-1316.

23.25. Burkhart SS, Esch JC, Jolson RS. The rotator crescent and rotator cable: an anatomic description of the shoulder's "suspension bridge". Arthroscopy 1993; 9: 611616.

24.26. Denard PJ, Koo SS, Murena L, Burkhart SS. Pseudoparalysis: the importance of rotator cuff integrity. Orthopedics 2012; 35: 1353-1357. 
25.27. Toussaint B, Audebert S, Barth J, et al; French Arthroscopy Society (SFA).

Arthroscopic repair of subscapularis tears: preliminary data from a prospective multicenter study. Orthop Traumatol Surg Res 2012; 98: S193-200.

550
26.28. Bartl C, Kouloumentas P, Holzapfel K, Eichhorn S, Woertler K, Imhoff A, Salzmann GM. Long-term outcome and structural integrity following open repair of massive rotator cuff tears. Int J Shoulder Surg 2012; 6: 1-8.

\subsection{Heikenfeld R, Gigis I, Chytas A, Listringhaus R, Godolias G. Arthroscopic} reconstruction of isolated subscapularis tears: clinical results and structural integrity after 24 months. Arthroscopy. 2012;28:1805-1811.

28.30. Nové-Josserand L, Hardy M-B, Ogassawara RLN, Carrillion Y, Godenèche A. Clinical and structural results of arthroscopic repair of isolated subscapularis tear. $\mathrm{J}$ Bone Joint Surg Am 2012; 94: e125.

\subsection{Lanz U, Fullick R, Bongiorno V, Saintmard B, Capemns C, Lafosse L.} Arthroscopic repair of large subscapularis tendon tears: 2- to 4-year clinical and radiographic outcomes. Arthroscopy 2013; 29: 1471-1478.

\subsection{Gerber C, Schneeberger AG, Hoppeler H, Meyer DC. Correlation of atrophy} and fatty infiltration on strength and integrity of rotator cuff repairs: a study in thirteen patients. J Shoulder Elbow Surg 2007; 16: 691-696. 
31.33. Oh JH, Kim SH, Choi JA, Kim Y, Oh CH. Reliability of the grading system for fatty degeneration of rotator cuff muscles. Clin Orthop Relat Surg 2010; 468(6): 15581564.

32.34. Jo $\mathrm{CH}$, Shin JS. Changes of appearance of fatty infiltration and muscle atrophy of rotator cuff muscles on magnetic resonance imaging after rotator cuff repair: establishing new time-zero traits. Arthroscopy 2013; 29: 449-458. 
Fig 1. Left shoulder, viewed through the dorsal A portal. SSC tendon with applied anchors and sutures before final reduction and knot tying with U-stitches for the medial row (black arrow). The remaining suture of the LHBT tenodesis was applied in a lasso-loop fashion at the upper border of the SSC lateral to the other SSC anchors (white arrow) in a pseudodouble-row configuration. $\mathrm{HH}$; humeral head.

Fig 2. (A) Right shoulder, viewed through the lateral $C$ portal. A cleavage lesion of the ISP/SSP tendon was reconstructed in a deep-layer lasso-loop technique ${ }^{14}$ for the medial row in order to achieve an anatomical reconstruction of the footprint.

(B) Schematic drawing showing the deep-layer lasso-loop technique for the reconstruction of rotator cuff tears with cleavage lesions. A smaller bite of the deep layer (d) is taken for the deep-layer lasso-loop followed by a larger bite of the superficial layer (D) with the same strand. With the second strand both layers are directly perforated, again a larger bite is taken for the superficial layer in a similar fashion. When the knot is tied the lasso-loop provides good pressure of the tendon onto the footprint for the deep layer and an anatomical reduction between the two layers towards each other. The footprint of the superficial layer is then reconstructed with the suture bridge technique.

Fig 3. Paracoronal MR views at one year of follow-up were analyzed to assess SSP tendon healing. (A) This example shows an intact footprint with complete healing of the SSP tendon (white arrow).

(B) Corresponding image demonstrating intratendinous signal abnormalities within the footprint of the SSP (white arrow). This repair was classified as intact as well. 
605 (C) Failed repair with complete rupture and retraction of the SSP tendon Patte II (white 606 arrow).

607

608 Fig 4. Axial MR images at one year follow-up for the assessment of the SSC footprint. (A)

609 Complete tendon healing with intact footprint (white arrow).

610 (B) Failed repair of the upper third of the SSC tendon with retraction (white arrow).

611 
613

614

615

616

629

630

631

632

633

634

635

\section{Preoperative $\quad$ Follow-up $\quad \mathrm{p}$ Value}

Strength

Modified Belly-press test (max. 5 points) $\quad 3.0 \pm 0.5(2-4) \quad 4.9 \pm 0.2(4-5) \quad<0.001$

Modified lift-off test (max. 5 points) $\quad 3.2 \pm 0.6(2-4)^{*} \quad 4.8 \pm 0.3(4-5) \quad<0.001$

Abduction strength test (max. 5 points) $\quad 2.9 \pm 0.4(2-4)^{* *} \quad 4.6 \pm 0.5(3-5) \quad<0.001$

External rotation strength (max. 5 points) $\quad 3.4 \pm 0.8(2-5) \quad 4.8 \pm 0.4(3-5) \quad<0.001$

Constant-Murley score

Total (maximum 100 points)

$36 \pm 16(16-64) \quad 86 \pm 12(50-100) \quad<0.001$

Pain (15 points)

$4.2 \pm 3.7(0-10) \quad 13.7 \pm 2.7(5-15) \quad<0.001$

Activity level (10 points)

$2.7 \pm 2.4(0-6) \quad 8.9 \pm 2.3(1-10) \quad<0.001$

$5.9 \pm 2.0(2-10) \quad 9.5 \pm 1.0(6-10) \quad<0.001$

$6.2 \pm 2.5(2-10) \quad 9.7 \pm 0.7(8-10) \quad<0.001$

$5.5 \pm 2.2(2-10) \quad 9.4 \pm 1.4(6-10) \quad<0.001$

$4.0 \pm 2.3(0-8) \quad 7.4 \pm 2.2(2-10) \quad<0.001$

$5.5 \pm 2.4(2-10) \quad 8.9 \pm 1.5(6-10) \quad<0.001$

$1.7 \pm 2.4(0-8) \quad 18.3 \pm 4.9(8-25) \quad<0.001$

Note: Data are given as mean \pm standard deviation (range). * 14 patients, ${ }^{*} 25$ patients. 
637

$$
\text { Fatty infiltration }
$$

$1.96 \pm 0.82(0-3)$

642

$$
\operatorname{CSA}\left(\mathrm{mm}^{2}\right)
$$

$1665 \pm 682(850-3140)$

$\begin{array}{cc}1.36 \pm 0.99(0-4) & <.0 .05 \\ 2026 \pm 638(670-3280) & <0.05\end{array}$

643 SSP

644

(n)

Fatty infiltration

$2.08 \pm 93(0-3)$

$1.48 \pm 92(0-3) \quad<0.05$

645

CSA $\left(\mathrm{mm}^{2}\right)$

488 \pm 164 (240-790)

$587 \pm 167(320-930) \quad<0.05$

646 ISP

647

$$
\text { Fatty infiltration }
$$

$1.04 \pm 0.82(0-3)$

$0.96 \pm 0.93(0-3)$

NS

648

CSA $\left(\mathrm{mm}^{2}\right)$

$1395 \pm 396$ (860-2340)

$1396 \pm 270$ (960-2000)

NS

649

650

651

Note: Data are given as mean \pm standard deviation (range). Fatty infiltration of the SSC/SSP/ISP was graded according to Goutallier et al. ${ }^{12}$, modified by Fuchs et al.

652

653

654 1820. The CSA of the SSC/SSP/ ISP were measured according to the method proposed by Zanetti et al. ${ }^{1921}$.

Abbreviation: CSA, cross-sectional area; SSC, subscapularis muscle; SSP, supraspinatus muscle; ISP, infraspinatus muscle; NS, not significant. 